\title{
Comparison of Rolling Moment Characteristics During Roll Oscillations for a Low and a High Aspect Ratio Configuration
}

\author{
Jay M. Brandon*, John V. Foster ${ }^{*}$, and Gautam H. Shah ${ }^{\dagger}$ \\ NASA Langley Research Center, Hampton, VA \\ and \\ William Gato ${ }^{\ddagger}$ and James E. Wilborn ${ }^{\S}$ \\ The Boeing Company - Commercial Airplanes Group, Seattle, WA
}

\begin{abstract}
Improvements in testing and modeling of nonlinear and unsteady aerodynamic effects for flight dynamics predictions of vehicle performance is critical to enable the design and implementation of new, innovative vehicle concepts. Any configuration which exhibits significant flow separation, nonlinear aerodynamics, control interactions or attempts maneuvering through one or more conditions such as these is, at present, a challenge to test, model or predict flight dynamic responses prior to flight. Even in flight test experiments, adequate models are not available to study and characterize the complex nonlinear and time-dependent flow effects occurring during portions of the maneuvering envelope. Traditionally, airplane designs have been conducted to avoid these areas of the flight envelope. Better understanding and characterization of these flight regimes may not only reduce risk and cost of flight test development programs, but also may pave the way for exploitation of those characteristics that increase airplane capabilities. One of the hurdles is that the nonlinear/unsteady effects appear to be configuration dependent. This paper compares some of the dynamic aerodynamic stability characteristics of two very different configurations - representative of a fighter and a transport airplane - during dynamic bodyaxis roll wind tunnel tests. The fighter model shows significant effects of oscillation frequency which are not as apparent for the transport configuration.
\end{abstract}

\section{Nomenclature}

$b \quad=$ reference wing span

$C_{L} \quad=$ lift coefficient

$C_{l} \quad=$ rolling moment coefficient

$C_{l p} \quad=$ roll damping parameter, $C_{l p}=C_{l p_{\text {body }}}+C_{l_{\dot{\beta}}} \sin (\alpha)$

$C_{l p_{\text {body }}}=$ body-axis roll damping derivative, $\frac{\partial C_{l}}{\partial\left(\frac{p_{b o d y} b}{2 V}\right)}$

$C_{l_{\beta}} \quad=$ body-axis sideslip derivative

\footnotetext{
*Aerospace Technologist, Vehicle Dynamics Branch, MS 153, Associate Fellow.

${ }^{\dagger}$ Aerospace Technologist, Vehicle Dynamics Branch, MS 153, Senior Member.

* Associate Technical Fellow, Aerodynamics - Stability and Control, P.O. Box 3707, MS 67-65

$\S$ Aerodynamics Engineer, Aerodynamics - Stability and Control, P.O. Box 3707, MS 67-62
} 


$\begin{aligned} C_{l_{\dot{\beta}}} & =\text { body-axis sideslip rate derivative, } \frac{\partial C_{l}}{\partial\left(\frac{\dot{\beta} b}{2 V}\right)} \\ k & =\text { reduced frequency, } k=\omega b / 2 V \\ p & =\text { roll rate } \\ \hat{p} & =\text { reduced roll rate, } \hat{p}=p b / 2 V \\ V & =\text { true airspeed } \\ \alpha & =\text { angle of attack } \\ \beta & =\text { angle of sideslip } \\ \phi & =\text { body-axis roll angle } \\ \omega & =\text { oscillation frequency }\end{aligned}$

\section{Introduction}

Improved prediction of airplane aerodynamic characteristics is required to improve design performance and safety, while at the same time reducing development and certification costs. Missed predictions of aerodynamic effects on flight dynamics and handling qualities of airplanes continue to give rise to large schedule impacts and costs when discovered in flight test. Aviation history is replete with such examples during the development of nearly every tactical fighter airplane ${ }^{1}$. Usually the large discrepancies between predicted and actual characteristics discovered in flight occur in non-linear or maneuvering flight regimes, such as high angles of attack, transonic maneuvering, or high angular rate maneuvering . For commercial airplanes, it is becoming more important to predict flying qualities in off-nominal flight conditions as well. Improved predictions may enable better departure resistance and recovery strategies, and may provide for higher fidelity pilot training in unusual flight conditions. Enhanced aerodynamic models for extreme envelope conditions will also improve analysis of airplane accidents and incidents, thereby providing a basis for improving safety of the airplane fleet.

One of the primary areas in which aerodynamic models are known to be deficient is in their response to high dynamic rates. Traditionally, dynamic rate effect models have consisted of coefficients that are linear functions of rate as obtained, classically in derivative form, from forced oscillation testing, and coefficients that are nonlinear functions of wind-axes axes roll rate as obtained from rotary balance testing. The resulting aerodynamic model structure computes the forces and moments based on instantaneous values of angular rates and the flow incidence angles, $\alpha$ and $\beta$. Forced oscillation results for many configurations often show large variation in the linear damping derivatives with oscillation amplitude and frequency, especially near stall angles of attack. These variations are indicators of either nonlinear rate effects or unsteady aerodynamic effects. Over the past few years as data handling and storage capabilities have improved, the time history data of complete forced oscillation cycles have been used for analysis, providing far more information than just the traditional linear derivatives. This research has led to improved understanding of the nonlinear and time-dependent effects present during unsteady airplane motion. In turn this has led to the development of higher fidelity modeling capabilities of airplane flight dynamics ${ }^{3-6}$.

Unfortunately, there appears to be a large variation in aerodynamic response depending on the airplane configuration tested, which adds to the difficulty of developing a proper physics-based model to describe airplane dynamic characteristics. This paper will compare the aerodynamic response to roll oscillations from low-speed forced oscillation wind tunnel tests of two very different configurations: a high-aspect ratio configuration representing a modern transport airplane, and a low-aspect-ratio configuration representing a modern fighter airplane prototype. Both sets of data were measured on the same testing apparatus and in the same wind tunnel using the same data reduction algorithms. Although data were obtained during oscillations in all three axes (pitch, roll, yaw), only roll axis data will be presented here. Additionally, many configuration variations were tested including control surface deflections, component build-ups, etc.; however, only one configuration for each airplane type will be presented.

\section{Wind Tunnel Tests}

Sketches of the two configurations tested are shown in Figs. 1 and 2. The fighter model (Fig. 1) features a blended wing/body with an aspect ratio of 2.1, twin vertical tails and no horizontal tails. The transport model (Fig. 2) is a twin-engine configuration and has a slender fuselage, slender wings with an aspect ratio of 7.9 and a conventional horizontal and vertical tail arrangement. Key configuration details are listed in Table 1. 
Table 1. - Geometry Comparison of Models

\begin{tabular}{|l|c|c|}
\hline \multicolumn{1}{|c|}{ Parameter } & Transport Model & Fighter Model \\
\hline Wing Span, $\mathrm{ft}$ & 6.85 & 5.40 \\
\hline Reference Wing Area, $\mathrm{ft}^{2}$ & 5.90 & 13.95 \\
\hline Aspect Ratio & 7.9 & 2.1 \\
\hline Mean Aerodynamic Chord, $\mathrm{ft}$ & 0.92 & 3.3 \\
\hline
\end{tabular}

The fighter model is shown mounted in the NASA Langley Research Center's 14x22-Foot Subsonic Wind Tunnel in Fig. 3. The transport model is shown mounted in the same wind tunnel in Fig. 4. Data were taken over a wide range of conditions, including angles of attack from $\pm 90^{\circ}$, oscillation amplitudes from $\pm 5^{\circ}$ to $\pm 30^{\circ}$, and oscillation frequencies from 0.23 to $1.10 \mathrm{~Hz}$, resulting in reduced frequencies $(k=\omega b / 2 \mathrm{~V})$ of $k=0.054$ to $k=0.455$. Table 2 lists the range of data obtained that will be presented in this paper.

Table 2. - Data Envelopes for Comparisons

\begin{tabular}{|l|c|c|}
\hline \multicolumn{1}{|c|}{ Parameter } & Transport Model & Fighter Model \\
\hline Amplitudes of oscillation & $0, \pm 5^{\circ}, \pm 10^{\circ}, \pm 20^{\circ}, \pm 30^{\circ}$ & $0, \pm 5^{\circ}, \pm 15^{\circ}, \pm 30^{\circ}$ \\
\hline Oscillation Frequency, Hz & $0.23-0.92$ & $0.3-1.10$ \\
\hline Reduced Frequency, $k$ & $0.054-0.216$ & $0.055-0.455$ \\
\hline Dynamic Pressure, $\mathrm{psf}$ & 10 & $2,5,10$ \\
\hline Angle of attack & $0^{\circ}-90^{\circ}$ & $-5^{\circ}-75^{\circ}$ \\
\hline$|\hat{p}|$ maximums during oscillations & $0.018-0.113$ & $0.005-0.238$ \\
\hline
\end{tabular}

The aerodynamic components of the dynamic data were obtained by first oscillating the model at set frequencies and amplitudes with the wind off to obtain gravity and inertial tare data and then by repeating the process with the wind on and subtracting the tare data. During both the wind-on and wind-off runs, data were obtained over 40 oscillation cycles, sampled at $200 \mathrm{~Hz}$ with $100 \mathrm{~Hz}$ anti-aliasing filters. The resulting data were then further filtered with a 4-Hz lowpass filter to remove unwanted frequency content. The dynamic data could then be ensemble averaged to represent the variation in aerodynamic forces and moments over a single oscillation cycle. Concurrently, the traditional calculations of damping derivatives using the in-phase and out-of-phase components of the balance outputs were calculated at the conclusion of each set of oscillations cycles. The computed damping derivatives actually combine roll-rate-damping and sideslip-rate effects in one single parameter, $C_{l p}=C_{l p_{b o d y}}+C_{l_{\beta}} \sin (\alpha)$. In addition to the dynamic data, complementary static wind tunnel data were obtained on the same test rig for comparison.

An indication of the "linear" characterization of forced oscillation data can be gained by inspection of the moment coefficient vs. oscillation angle. A conventional modeling approach $C_{l}=C_{l_{\phi}} \phi+C_{l_{p}} \hat{p}$ yields an inclined oval as depicted in Fig. 5. The inclination of the oval represents the static stability, or restoring moment due to roll angle (or sideslip) and the vertical diameter of the oval represents the damping effect, or restoring moment due to angular rate. Deviations from this oval shape indicate non-linear effects that cannot be modeled using the summation of linear, first-order derivatives. In order to capture these non-linear effects, higher order functions or table lookups are often required.

\section{Fighter Model Data Results}

Static data (Fig. 6) show maximum lift for the fighter model occurs at approximately $\alpha=34^{\circ}$. with an earlier decrease in the lift curve slope noticed at $\alpha=24^{\circ}$. Static rolling moment characteristics (Fig. 7) show a large asymmetry which also occurs near $\alpha=24^{\circ}$. This combination of characteristics would certainly warrant further investigation as to the mechanisms and dynamic characteristics if flight near this angle of attack was anticipated. Figure 8 shows the classical results from forced oscillation testing. For this example, forced oscillation tests with an oscillation amplitude of $\pm 30^{\circ}$ were conducted at a number of reduced frequencies, $k$. The test data show that at low $\alpha$ 's, roll damping $\left(C_{l p}\right)$ is relatively invariant with oscillation frequency suggesting that the roll damping parameter is also apparently invariant across the range of roll rates tested, since at a given amplitude, varying frequency also 
varies the maximum roll rates generated. At higher angles of attack, the data is not as well behaved as evidenced by large variations of the roll damping parameter with frequency from approximately $\alpha=24^{\circ}$ to $\alpha=40^{\circ}$. This characteristic behavior has been observed in tests for decades, and until recently, has only been addressed by selecting model test frequencies near full-scale airplane response frequencies of interest (i.e. dutch-roll frequency for example), or by parametrically varying roll damping across a wide range of potential values to ensure sufficient robustness in the flight control system design.

More recently, testing and analysis has been conducted in an attempt to reduce the uncertainty in the dynamic characteristics measured in forced oscillation tests. An alternative data reduction approach, called the single-point method, uses data obtained from time history recordings of the forced oscillation cycles. This method uses instantaneous values of the aerodynamic forces and moments measured as the model moves through the midpoint of the sinusoidal rolling motions where the oscillation amplitude is zero, $\beta=0^{\circ}$, rotation rate is a maximum, and there is no rotational acceleration. The value of the aerodynamic forces and moments at these points (one at a positive rate, and one at a negative rate) can then either be divided by the non-dimensional rate at each of the points to obtain a derivative, or can be plotted versus rate to develop a nonlinear dynamic model that is a function of rate The variation of nondimensional roll rate can be accomplished by changing either the frequency or the amplitude of oscillation. To maintain constant reduced frequencies for rate variations, both oscillation frequencies and amplitudes must be varied. The test technique is limited to variations of two of the critical parameters ( $\hat{p}, k$, amplitude) in order to keep the other constant. Therefore, amplitude effects - due to transitions from one flow-field state to another during the oscillation - and frequency effects, which are an indication of unsteady aerodynamic phenomena, may be difficult to isolate.

Figures 9-11 show the rolling moment characteristics as the model is oscillated. The cases were selected such that each data set was measured at the same nondimensional roll rate $(\hat{p}=0.04)$ as the model rolled through the midpoint of the oscillation, at $\phi=0^{\circ}$. For a linear, symmetric model of the roll damping to be valid, data from each amplitude of oscillation should coincide at the $\phi=0^{\circ}$ point, since the states $(\alpha, \beta, \hat{p})$ are identical for each data set. At $\alpha=10^{\circ}$ (Fig. 9), well before stall where the aerodynamic responses are primarily linear, the data all coalesce at $\phi=0^{\circ}$ regardless of amplitude of oscillation. As $\alpha$ is increased to $\alpha=30^{\circ}$ (Fig. 10), near the stall angle of attack, the data loops do not coalesce at $\phi=0^{\circ}$, and show that as the oscillation amplitude increases, there is an apparent increase in roll damping. At post-stall $\alpha$ 's (Fig. 11), again the data do not coalesce at $\phi=0^{\circ}$, however the smaller amplitude data show higher apparent damping for this condition. The observed nonlinearity of the results at the stall and post-stall $\alpha$ conditions indicate a higher order model is needed to describe the data.

Figures 12-14 show single-point data versus nondimensional roll rate. The plots include data obtained from a range of oscillation amplitudes and frequencies, at several wind tunnel velocities. Typically, three points along the $p b / 2 V$-axis are plotted for each reduced frequency, each representing different oscillation amplitudes, to show the variation with rate at a given reduced frequency. Previous comparisons of the dynamic roll data have shown tunnel velocity effects to be insignificant, with reduced frequency and rate being the predominant effects. The data in Fig. 12 show that at $\alpha=10^{\circ}$, the rolling moment variation with roll rate is slightly nonlinear, but apparently no significant reduced frequency effects are evident. At $\alpha=30^{\circ}$ (Fig. 13), there is a clear indication of the variation in the dynamic stability characteristics with reduced frequency. These data correlate well to the data in Fig. 8, which also show variations in roll damping with reduced frequency. At very high $\alpha$ 's, the data show pronounced nonlinearities in both reduced-frequency and roll-rate effects as seen in Fig. 14 at $\alpha=50^{\circ}$. The data in Fig. 14 show roll damping is unstable at low rates, and stable at high rates. This roll damping characteristic can give rise to wing rock type motions if the static stability $\left(C_{l_{\beta}}\right)$ provides the required "spring action" to the dynamic system.

\section{Transport Model Data Results}

The transport model was tested in a flaps-up cruise configuration. Static data (Fig. 15) show initial flowseparation wing stall occurring around $\alpha=10^{\circ}$, followed by a second post-stall increase in planform lift up to a maximum at approximately $\alpha=40^{\circ}$. The flow separation-induced stall manifests itself as a discontinuity in the lift curve slope, and experience has shown that such discontinuities are focus points for further analysis of the stability characteristics of the configuration. 
Figure 16 shows the static rolling moment characteristics of the model at $\beta=0^{\circ}$. Roll asymmetries are small below $\alpha=40^{\circ}$, then build slowly to a large peak between $\alpha=40^{\circ}$ and $\alpha=60^{\circ}$. Limited flow visualization studies conducted on the transport model suggest that the likely cause of this peak is due to interactions between two vortex sheets that roll up over the fuselage and the inboard wings/vertical tail at high $\alpha$ 's. It is noteworthy that the wing/vortex interaction appeared to be significantly less than is often observed on fighter configurations - which is a possible explanation for the differences in frequency effects previously discussed. Further flow visualization or surface pressure measurements are warranted to further investigate these effects.

Figure 17 shows the classical results from forced oscillation tests conducted at several frequencies and amplitudes. The data show no appreciable effects from variations of either frequency or amplitudes up through $\alpha=20^{\circ}$. Above $\alpha=20^{\circ}$, a nonlinear variation of the computed derivative occurs, indicating nonlinear roll damping characteristics.

Figures 18-21 show the rolling moment variation as the model oscillates at three different combinations of amplitude and frequency while maintaining the same nondimensional maximum roll rate as the model passes through $\phi=0^{\circ}(\hat{p}=0.0188)$. All four figures are plotted at the same scale to facilitate comparisons. As with the fighter model, the data coalesce at $\alpha=0^{\circ}$ (Fig. 18) and resemble the ellipses indicative of linear variation of $C_{l_{\beta}}$ and $C_{l p}$. At $\alpha=10^{\circ}$ (Fig. 19), distortions in the elliptical shape of the data appear, and at $\alpha=12^{\circ}$ (Fig. 20) the ellipses practically collapse into a single curve, indicative of neutral damping, and exhibit slope changes that are amplitude dependent. At $\alpha=40^{\circ}$ (Fig. 21) the classical elliptical loops are significantly distorted, with a change in direction (twisting) of the loops at the larger amplitudes as indicated by the arrows showing the direction of the oscillations. This twisting of the loops at the large oscillation amplitudes is indicative of a change in the damping characteristics from unstable at the small amplitudes (highest rates) to stable at the larger amplitudes (lowest rates).

In general, the non-elliptical shapes of the rolling moment data at higher angles of attack highlight the inadequacy of the current linear mathematical models of roll damping. Figure 22 presents the classical forced oscillation results for the same data sets as shown in Figs. 18-21. The conventional approach of calculating the roll damping parameter involves integrating the out-of-phase (with roll angle) component of rolling moment over the entire oscillation cycle. This integration procedure results in an "average" value of the damping parameter over the oscillation cycle. The conventional results show unstable damping at the small amplitude oscillation at $\alpha=40^{\circ}$, and stable damping at the large amplitude oscillation. Inspection of the actual time-history data (Fig. 21) shows all three amplitudes of oscillation exhibit unstable damping characteristics at small roll angles.

The single-point approach predicts a well behaved, stable, and nearly linear variation of rolling moment with nondimensional roll rate at $\alpha=0^{\circ}$ (Fig. 23). At $\alpha=10^{\circ}$ (Fig. 24), the rolling moment variation with roll rate shows a slightly nonlinear behavior, and has a slightly lower slope, but is still well behaved and indicates stable roll damping. At $\alpha=12^{\circ}$ (Fig. 25), the variation of rolling moment with roll rate flattens or becomes slightly unstable. At $\alpha=40^{\circ}$, the single-point analysis (Fig. 26) indicates unstable roll damping at small rates and stable damping at higher roll rates. This is an indicator of potential wing rock characteristics ${ }^{7}$ at this angle of attack; however, more data is required at lower rates to fully describe the unstable region - particularly near $p b / 2 V=0$, where a large change in rolling moment is seen between the two adjacent points. Observations of the available data at low rates seen at the maximum roll angles during the oscillation cycles (Fig. 21) seem to show a stable damping effect. Unfortunately, at these roll angles, the combined rotational acceleration and sideslip effects may have an impact on the apparent damping characteristics which the single-point approach does not capture since the single-point approach includes data only from the mid-point of the oscillation cycle, where rotational acceleration and sideslip are zero.

\section{Data Summary and Conclusions}

The data obtained for the low aspect ratio fighter model show strong effects of frequency on the rolling moment response to the forced oscillation motions. These results illustrate not only the current limitations of modeling dynamic rate effects with the conventional forced oscillation derivative approach, but also of the more detailed single point approach, considering that the nonlinear rate effects that resulted from this approach still failed to adequately predict the observed characteristics. The strong dependence of the rolling moment characteristics on reduced frequency in the stall $\alpha$ range indicates that unsteady and/or nonlinear aerodynamic phenomena are important, and that new modeling approaches that account for these effects need to be developed. 
The conventional damping derivatives calculated from the high aspect ratio transport model dynamic data were not adequate to model the characteristics observed during testing. Further analysis showed that the dynamic characteristics were nonlinear with roll rate; however, the reduced frequency effects were not evident in the data at $\alpha$ 's below $20^{\circ}$. Simple nonlinear mapping of rolling moment coefficient with nondimensional roll rate worked well to characterize most of the responses measured in the test. The resulting model represents a vast improvement to the current state-of-the art in modern transport simulation and analysis techniques. However, work remains to be done in isolating and modeling rotational-acceleration and sideslip effects to better characterize the responses measured in the test.

Both configurations exhibited a requirement to move beyond linear damping derivatives in order to better model the aerodynamic effects measured in the roll oscillation tests. The high-aspect-ratio transport configuration showed nonlinear roll rate dependence, which is easily adapted into conventional look-up table approaches and can be included with only minor changes to simulation architecture. In stark contrast were the results for the low-aspect ratio fighter model. The data indicated not only nonlinear dependence on roll rate, but also strong dependence on reduced frequency. The variation in aerodynamic response with reduced frequency indicates the possible existence of time-dependent nonlinear behavior, which will require additional state variables in the mathematical models.

Further work should be conducted to identify the causes for different response characteristics between the two models. One likely candidate is the wing aspect ratio; however, many other factors such as sweep angle, chord length, tail planform and placement among others must also be evaluated. Work in developing math modeling techniques to fully describe airplane responses to dynamic motions needs to be pursued. Different motion shapes (other than sinusoidal oscillations) and effects of motions about other axes need to be explored as well in order to obtain a comprehensive data set over a wide range of conditions.

\section{References}

${ }^{1}$ Chambers, J.; and Hall, R.: Historical Review of Uncommanded Lateral-Directional Motions at Transonic Conditions. AIAA 2003-0590, January 2003.

${ }^{2}$ Chambers, Joeseph R.; and Grafton, Sue B.: Static and Dynamic Longitudinal Stability of a Powered 1/9-Scale Model of a Tilt-Wing V/STOL Transport. NASA TN D-3591, September 1966.

${ }^{3}$ Brandon, J.M.; and Foster, J.V.: Recent Dynamic Measurements and Considerations for Aerodynamic Modeling of Fighter Airplane Configurations. AIAA 98-4447, August 10-12, 1998.

${ }^{4}$ Murphy, P., and Klein, V.: Validation of Methodology for Estimating Aircraft Unsteady Aerodynamic Parameters form Dynamic Wind Tunnel Tests. AIAA 2003-5397, August 2003.

${ }^{5}$ Lan, C. Edward, Li, Jilu, Yau, Waipang, and Brandon, Jay M.: Longitudinal and Lateral-Directional Coupling Effects on Nonlinear Unsteady Aerodynamic Modeling From Flight Data. AIAA 2002-4804, August 2002.

${ }^{6}$ Goman, M.; and Khrabrov, A.: State-Space Representation of Aerodynamic Characteristics of an Aircraft at High Angles of Attack. AIAA 92-4651, 1992.

${ }^{7}$ Nguyen, L. T.; Yip, L. P.; and Chambers, J. R.: Self Induced Wing Rock of Slender Delta Wings. AIAA 811883, August 1981.

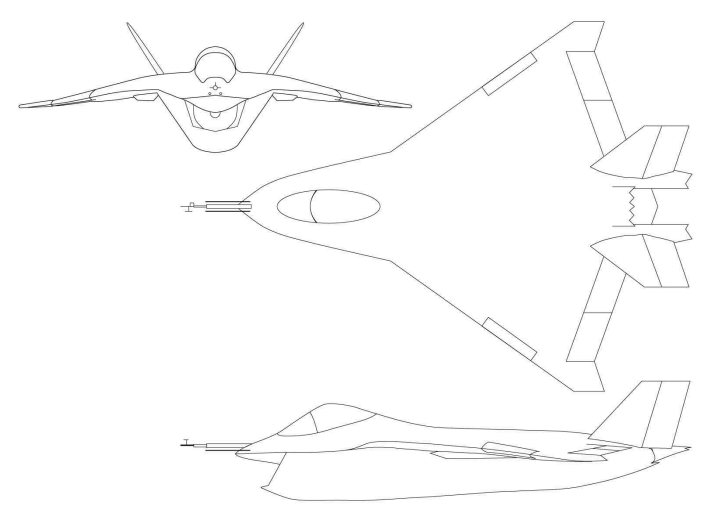

Figure 1. Sketch of the fighter model

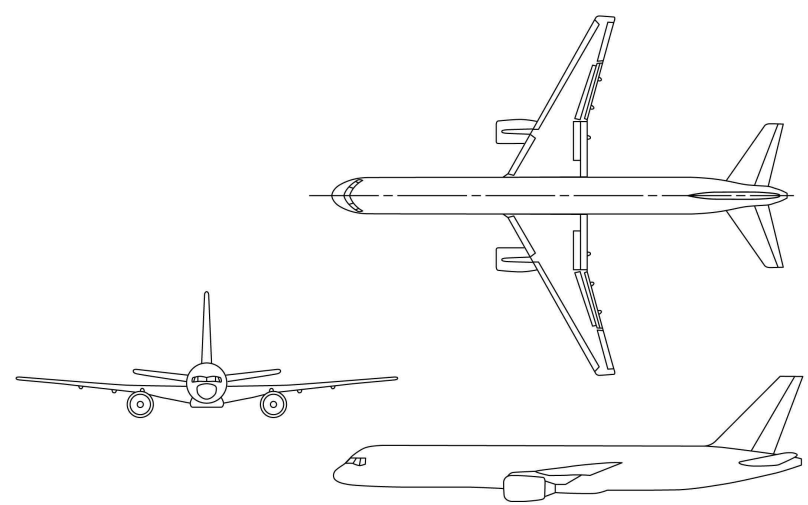

Figure 2. Sketch of the transport model 


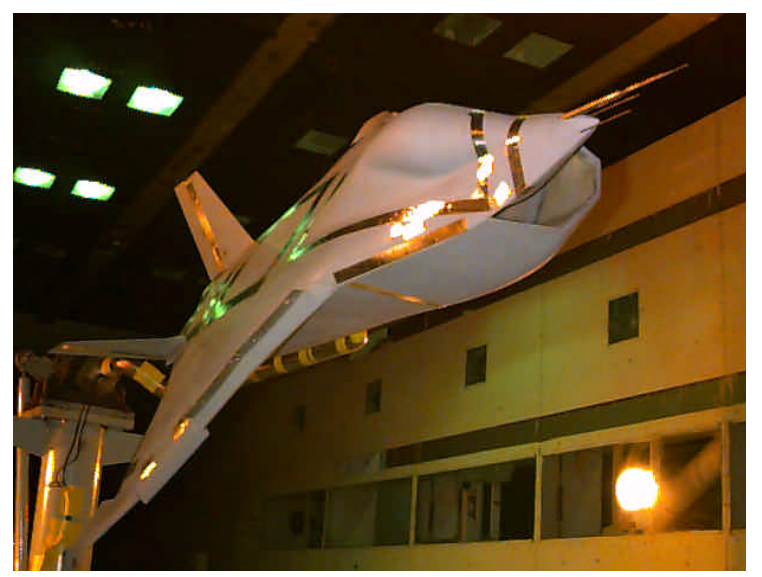

Figure 3. Fighter model mounted in wind tunnel for roll-oscillation tests.

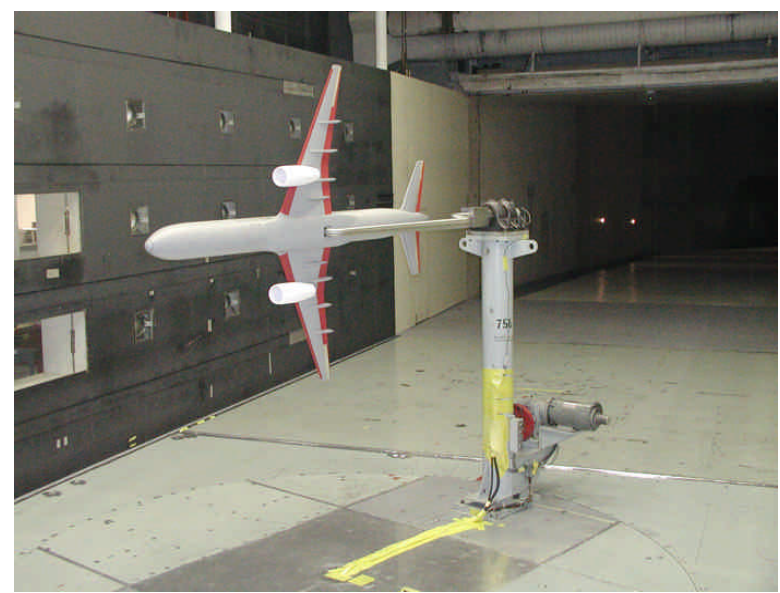

Figure 4. Transport model mounted in wind tunnel for roll oscillation tests.

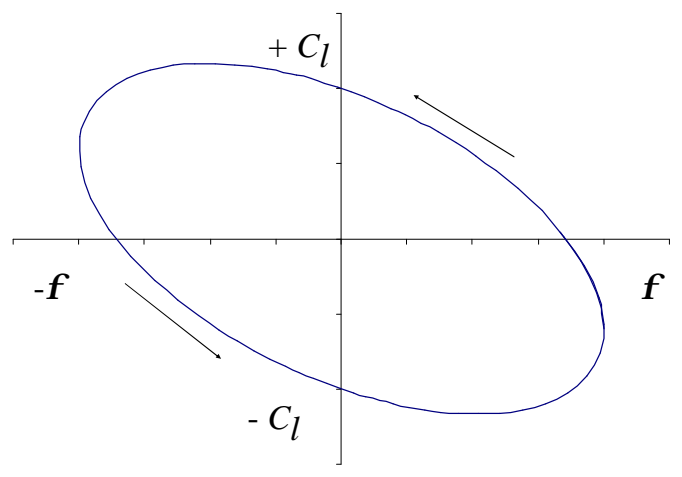

Figure 5. Characteristic shape for stable static and dynamic linear aerodynamics.

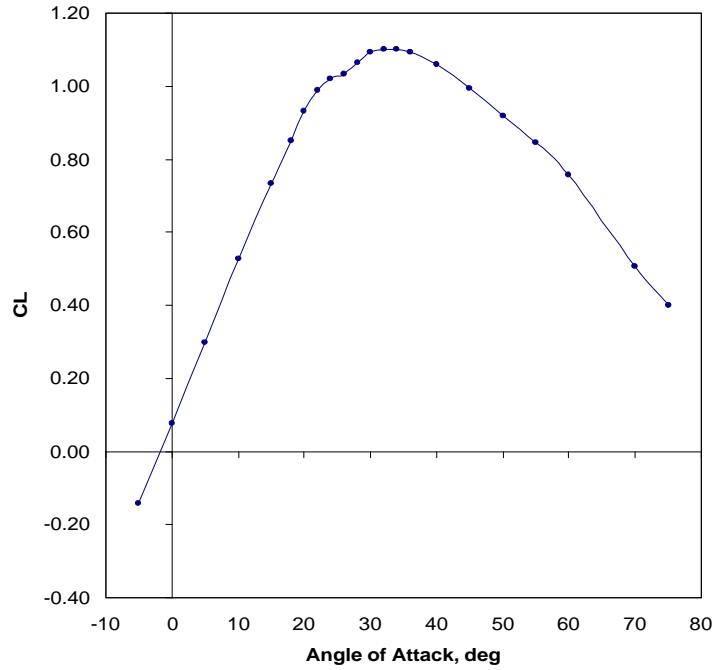

Figure 6. Static lift data for fighter model mounted on roll oscillation test system.

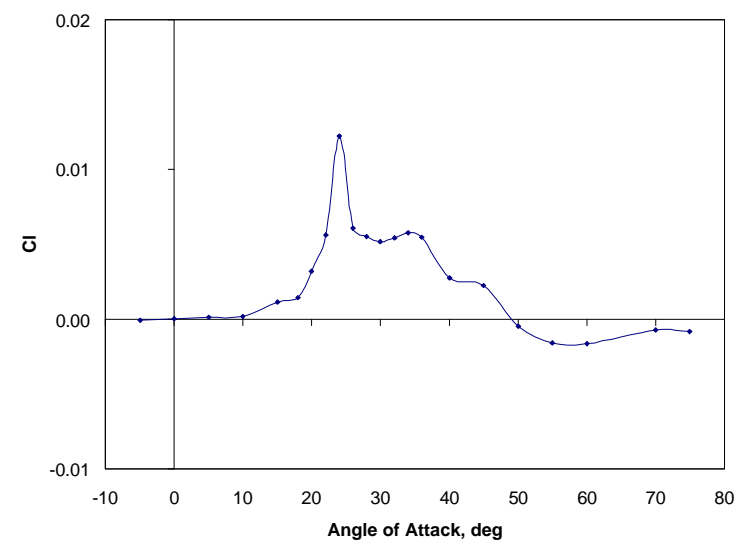

Figure 7. Fighter model rolling moment at $\beta=0^{\circ}$ on roll oscillation test system.

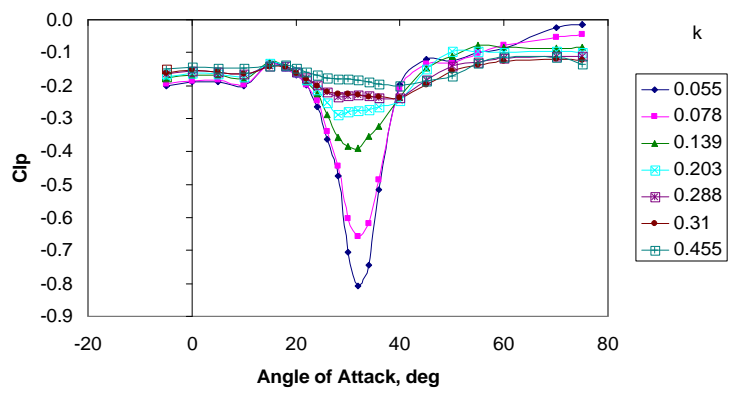

Figure 8. Conventional roll damping parameter for fighter model from roll oscillation tests at oscillation amplitude of $30^{\circ}$. 


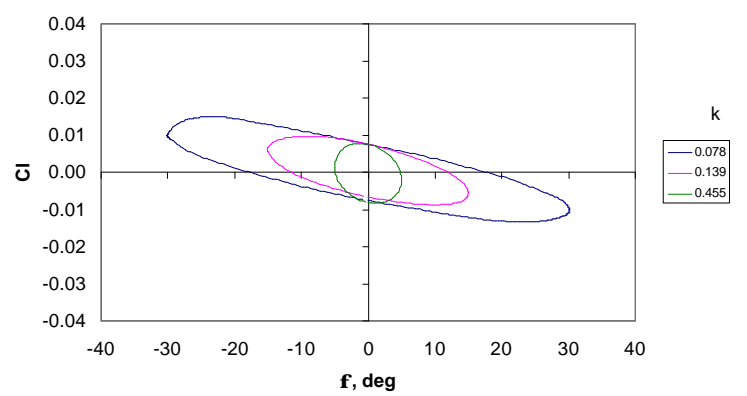

Figure 9. Rolling moment for the fighter model at $\alpha=10^{\circ}$ during oscillations with $\hat{p}_{\text {max }}=0.04$.

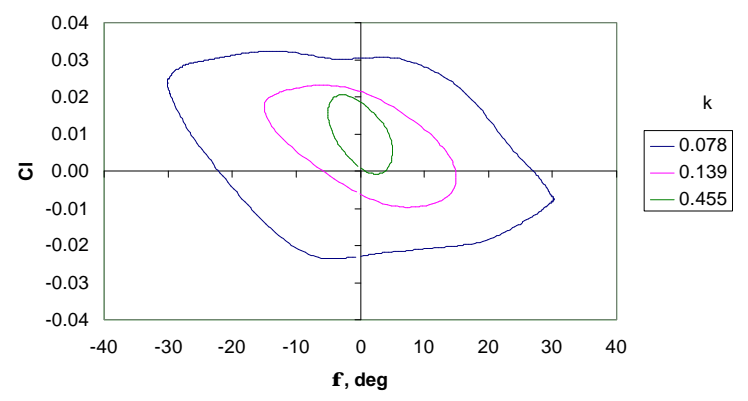

Figure 10. Rolling moment for the fighter model at $\alpha=30^{\circ}$ during oscillations with $\hat{p}_{\text {max }}=0.04$.

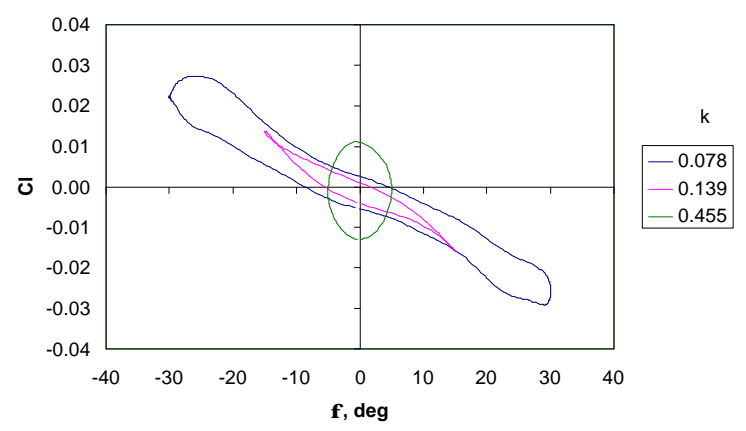

Figure 11. Rolling moment for the fighter model at $\alpha=50^{\circ}$ during oscillations with $\hat{p}_{\text {max }}=0.04$.

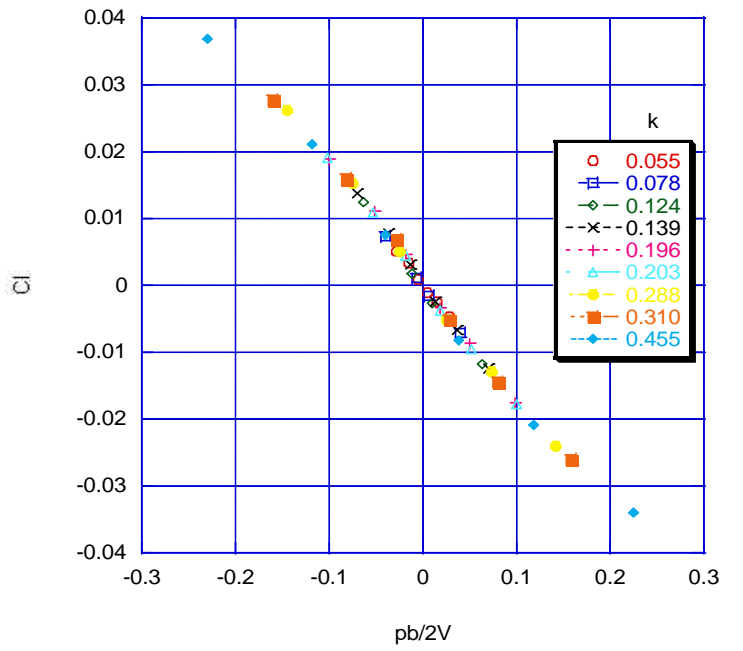

Figure 12. Effect of reduced frequency on rolling moment. Fighter model, $\alpha=10^{\circ}$.

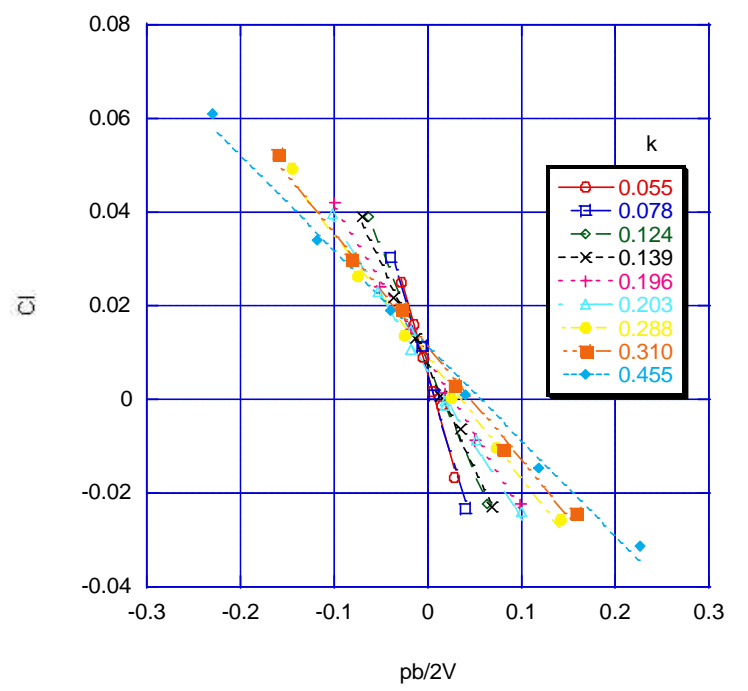

Figure 13. Effect of reduced frequency on rolling moment. Fighter model, $\alpha=30^{\circ}$. 


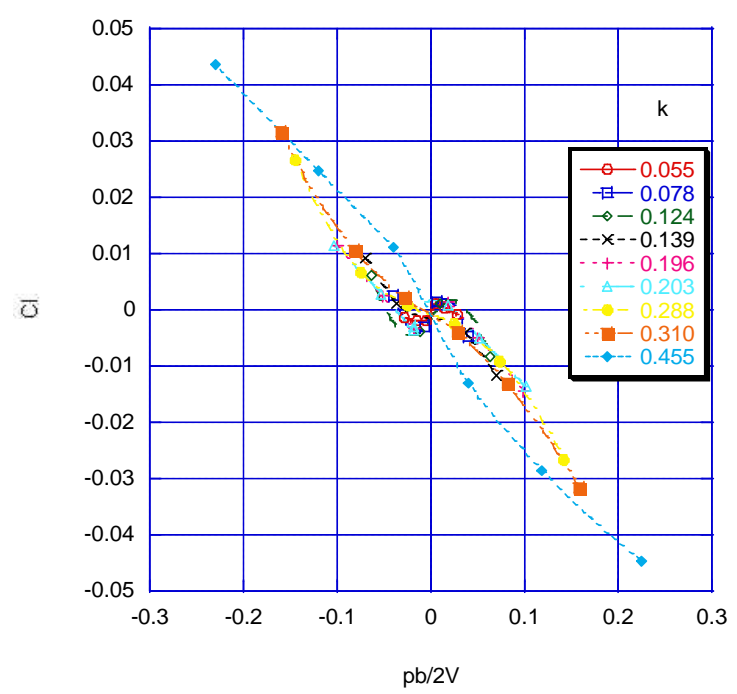

Figure 14. Effect of reduced frequency on rolling moment. Fighter model, $\alpha=50^{\circ}$.

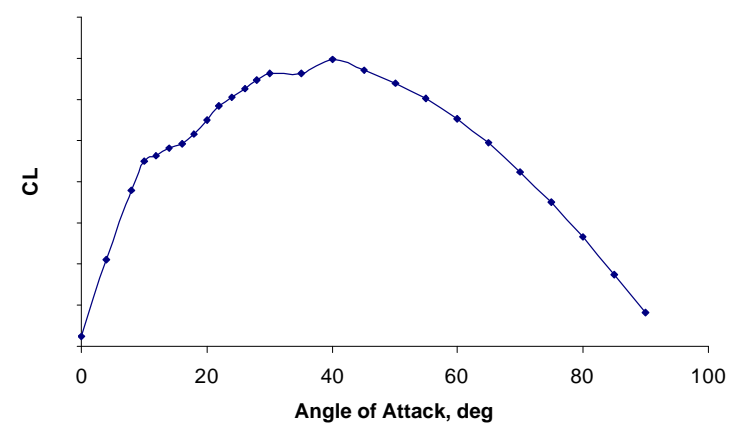

Figure 15. Static lift data for transport model mounted on roll oscillation test system.

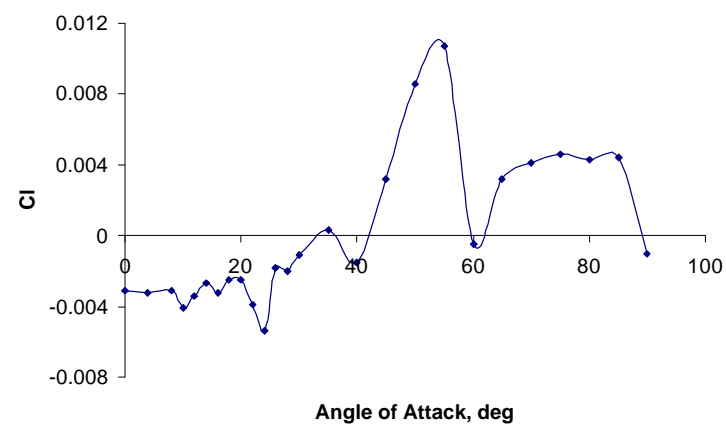

Figure 16. Transport model rolling moment at $\beta=0^{\circ}$ on roll oscillation test system.



Figure 17. Conventional roll damping parameter for transport model from roll oscillation tests at oscillation amplitude of $30^{\circ}$.

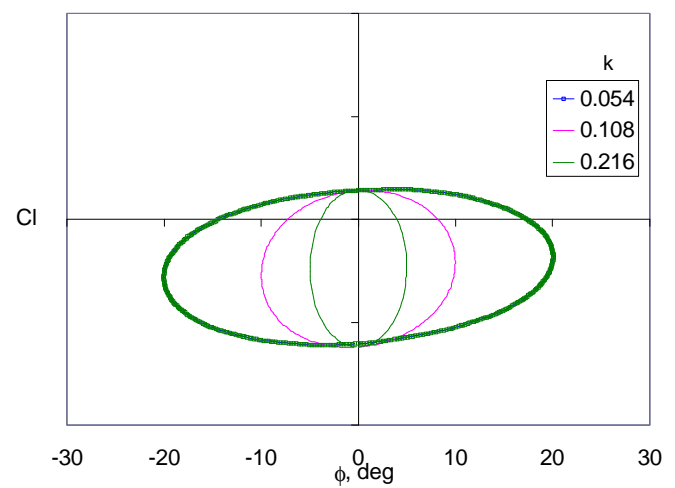

Figure 18. Rolling moment for the transport model at $\alpha=0^{\circ}$ during oscillations with $\hat{p}_{\text {max }}=0.0188$.



Figure 19. Rolling moment for the transport model at $\alpha=10^{\circ}$ during oscillations with $\hat{p}_{\text {max }}=0.0188$. 




Figure 20. Rolling moment for the transport model at $\alpha=12^{\circ}$ during oscillations with $\hat{p}_{\max }=0.0188$.

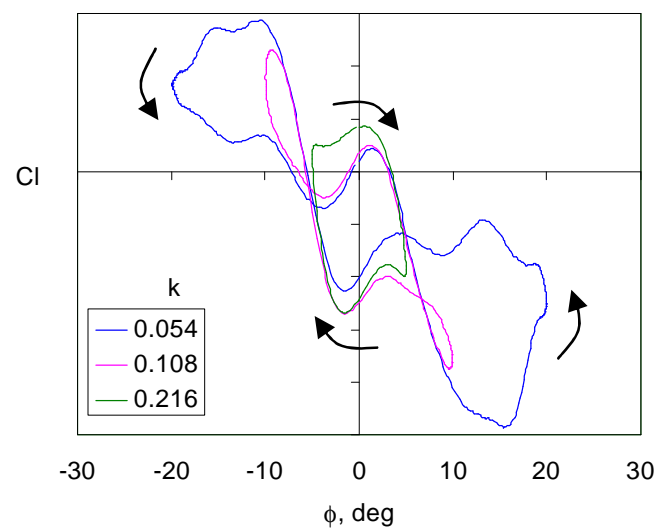

Figure 21. Rolling moment for the transport model at $\alpha=40^{\circ}$ during oscillations with $\hat{p}_{\max }=0.0188$.

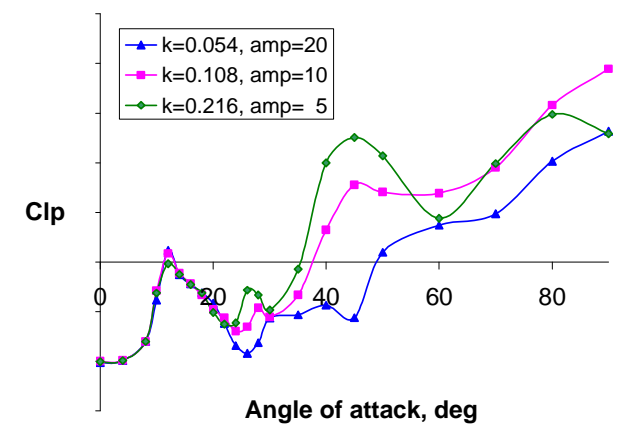

Figure 22. Conventional roll damping parameter for the transport model during oscillations with $\hat{p}_{\max }=$ 0.0188 .

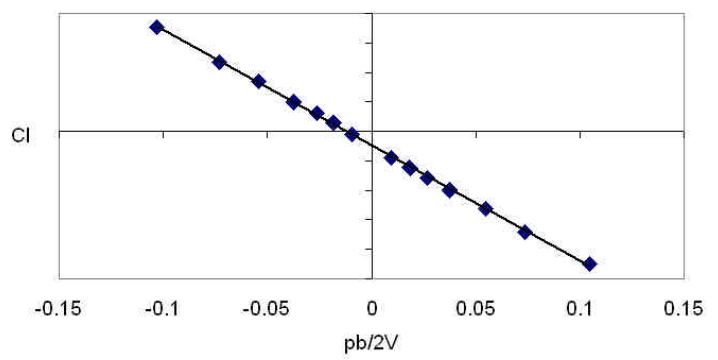

Figure 23. Single-point results for transport model at $\alpha=0^{\circ}$ and all frequency-amplitude combinations tested.

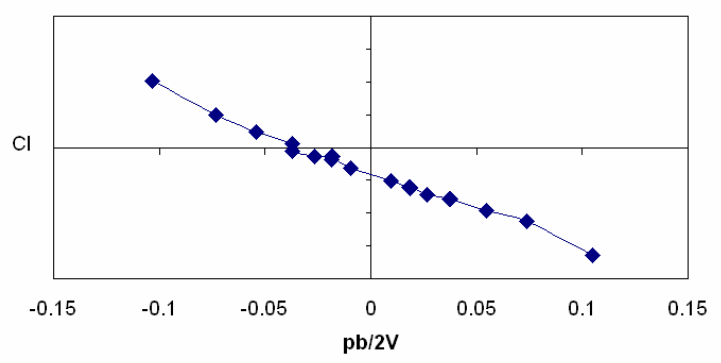

Figure 24. Single-point results for transport model at $\alpha=10^{\circ}$ and all frequency-amplitude combinations tested.

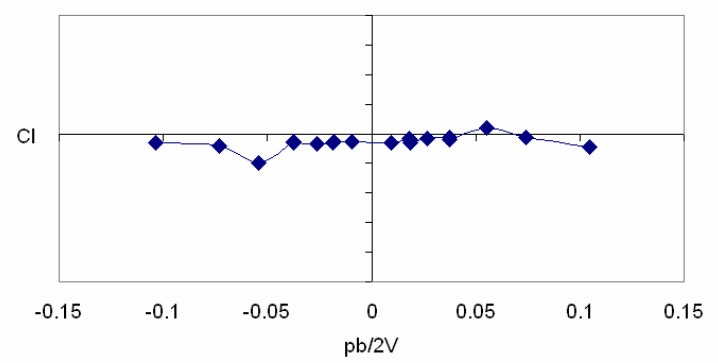

Figure 25. Single-point results for transport model at $\alpha=12^{\circ}$ and all frequency-amplitude combinations tested.

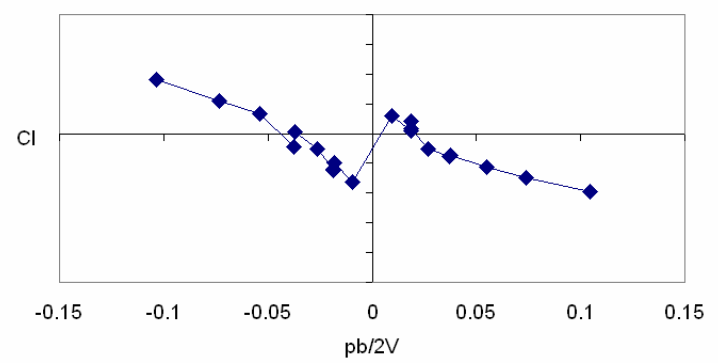

Figure 26. Single-point results for transport model at $\alpha=40^{\circ}$ and all frequency-amplitude combinations tested. 